\title{
Interactions with Existing and Potential Customers: The Role of Physical and Virtual Trade Fairs: An Abstract
}

\author{
Maria Sarmento and Cláudia Simões
}

\begin{abstract}
Trade fairs are recognized as important platforms for product promotion and new sales or lead generation (Kerin and Cron 1987) and stimulation of reflexive practices within firms (Bathelt and Schuldt 2008). Trade fairs may use physical platforms, where participants interact face-to-face, or virtual platforms, where participants interact through computer-mediated forms. This study constitutes a preliminary attempt to understand the association between physical and virtual trade fairs. The fieldwork combines qualitative interviews with trade fair organizers, exhibitors, and visitors and a survey comprised of open-ended questions administrated to visitors of an international trade fair. The main conclusion is that trade fairs are instrumental and relevant platforms for interaction and customer engagement processes. The physical trade fair is core to raise the proximity among participants and allows the direct interaction with the product or service. The virtual trade fair emerges as a platform for communication, global market reach, and cost/time savings. Findings further support that physical and virtual platforms should be combined in an integrated and complementary way. This complementary effect brings the opportunity to capitalize on the strengths of both platforms. Ultimately, an optimal combination of the physical and virtual platforms may be pivotal in fostering learning and knowledge dissemination and exchange on a continuous basis, favoring practices for relationship marketing and customer engagement. Research implications and avenues for future studies are presented.
\end{abstract}

References Available Upon Request

\author{
M. Sarmento $(\square)$ \\ Nova School of Business and Economics, Lisboa, Portugal \\ e-mail: maria.sarmento@novasbe.pt \\ C. Simões \\ University of Minho, Braga, Portugal \\ e-mail: csimoes@eeg.uminho.pt
}

(C) Academy of Marketing Science 2019 